\title{
High-accuracy propagation of light rays
}

\author{
Pierre Teyssandier ${ }^{1}$ \\ ${ }^{1}$ Dépt SYstèmes de Référence Temps-Espace (SYRTE), CNRS-UMR 8630 \& UPMC, \\ Observatoire de Paris, 61 avenue de l'Observatoire, 75014 Paris \\ email: Pierre.Teyssandier@obspm.fr
}

\begin{abstract}
We present a review of the different methods currently developed to determine the deflection of light rays due to gravity. The aim of these methods is primarily to calculate the angular distances with an accuracy of the order of microarcsecond.
\end{abstract}

Keywords. Gravitation, astrometry, reference systems.

\section{Introduction}

Space astrometric missions like Gaia or SIM are aimed to reach an accuracy of the order of microarcsecond ( $\mu \mathrm{as}$ ) in the measurements of the positions of celestial objects. We give a review of the main methods which are at our disposal to treat the high-accuracy propagation of light in the framework of metric theories of gravity.

\section{Newtonian approach}

The idea that light might be deflected by a gravitating body has appeared a long time before Einstein. A Newtonian deflection $\widehat{\delta}_{N}$ was calculated for an unbound orbit in the field of an isolated, spherically symmetric body of mass $M$ around 1784 by Cavendish and then by Soldner in 1801 (see Will 1988). These old results are equivalent to the exact formula

$$
\sin \frac{\widehat{\delta}_{N}}{2}=\frac{G M}{V^{2} b}\left[1+\left(\frac{G M}{V^{2} b}\right)^{2}\right]^{-1 / 2},
$$

where $G$ is the gravitational constant, $V$ is the speed of light at infinity and $b$ the impact parameter of the ray. For a ray grazing the surface of the Sun, Eq. (2.1) yields approximatively half the value predicted by general relativity, that is $\widehat{\delta}_{N}=0.875$ arcsecond.

It may be concluded from Eq. (2.1) that the gravitational deflection of light should be taken into account in modern astrometry even if Newtonian theory was right.

\section{Modern, relativistic approach: metric theories}

Newton's theory is now replaced by the so-called relativistic metric theories. So we suppose henceforth that space-time $\mathcal{V}_{4}$ is endowed with a Lorentzian metric $g$ describing gravity. Recall that this metric enables to define the scalar product $X . Y$ of two vectors $X$ and $Y$ at a given point: in any coordinate system $\left(x^{\alpha}\right)$, one has $X . Y=g_{\alpha \beta} X^{\alpha} Y^{\beta}=$ $g^{\alpha \beta} X_{\alpha} Y_{\beta}$. We put $X^{2}=X . X=g_{\alpha \beta} X^{\alpha} X^{\beta}$. The signature chosen for $g$ is $(+,-,-,-)$.

At the $1 \mu$ as level of accuracy, the propagation of light may be analyzed within the geometric optics approximation. Then light rays are null geodesics of space-time (see Section 5). Recall that a curve is said to be null when its tangent vector $l^{\alpha}$ is a null 
vector, that is a vector such that

$$
l^{2}=g_{\alpha \beta} l^{\alpha} l^{\beta}=0 .
$$

Null geodesics were determined in a closed-exact form for Schwarzschild metric in de Jans (1922). However, this exact solution is expressed in terms of elliptic functions which are difficult to handle, so that only perturbation solutions are used in practice. The total deflection of a light ray coming from infinity with an impact parameter $b \gg 2 G M / c^{2}$ is given by

$$
\widehat{\delta}=\frac{4 G M}{c^{2} b}+\frac{15 \pi}{4}\left(\frac{G M}{c^{2} b}\right)^{2}+O\left(1 / c^{6}\right) .
$$

It must be noted that the validity of the formula (3.2) is independent of the coordinate system because the impact parameter is a length defined for an (ideal) observer at rest at infinity, where space-time is Minkowskian.

For a ray grazing the Sun, the term of order $c^{-4}$ in Eq. (3.2) amounts to $11 \mu$ as. Since the angle between the spacecraft rotation axis and the Sun direction is equal to $45 \mathrm{deg}$, the contribution of this term will remain completely unobservable by Gaia mission.

In what follows we examine how the gravitational deflection of light can be calculated for more general gravitational fields.

\section{Angular distances in metric theories}

Consider two light rays $\Gamma$ and $\Gamma^{\prime}$ arriving at a point $x_{o} \in \mathcal{V}_{4}$. We assume that $\Gamma$ and $\Gamma^{\prime}$ are emitted by point-like sources $\mathcal{E}$ and $\mathcal{E}^{\prime}$, respectively. Let $\mathcal{O}(u)$ be an observer passing through $x_{o}$ with a unit 4 -velocity $u$, i.e. the vector of components $u^{\mu}=d x^{\mu} / d s$.

Space relative to $\mathcal{O}(u)$ at $x_{o}$ is defined as the set $\Pi_{x_{o}}(u)$ of the tangent vectors orthogonal to the 4 -velocity of $\mathcal{O}(u)$. As a consequence, the angular distance between $\mathcal{E}$ and $\mathcal{E}^{\prime}$ as measured by $\mathcal{O}(u)$ is defined as the angle $\phi_{u}$ between the orthogonal projections of the rays $\Gamma$ and $\Gamma^{\prime}$ on $\Pi_{x_{o}}(u)$ (see Soffel 1989 or Brumberg 1991). Let $l$ and $l^{\prime}$ denote vectors tangent to $\Gamma$ and $\Gamma^{\prime}$ at $x_{o}$, respectively. Taking into account that $l$ and $l^{\prime}$ are null vectors, it may be seen that the angular distance $\phi_{u}$ is determined by the relation

$$
\sin ^{2} \frac{\phi_{u}}{2}=-\frac{\left(l^{\prime}-l\right)^{2}}{4(u . l)\left(u . l^{\prime}\right)}, \quad 0 \leqslant \phi_{u} \leqslant \pi .
$$

Two kinds of descriptions may be envisaged in modeling astrometric measurements.

1. One can introduce an orthonormal tetrad $e_{\underline{\alpha}}$ along the worldline of $O(u)$ such that

$$
e_{\underline{0}}=u, \quad u \cdot e_{\underline{i}}=0, \quad e_{\underline{i}} \cdot e_{\underline{j}}=-\delta_{i j}, \quad(i=1,2,3) .
$$

The direction of the ray $\Gamma$ is then defined by the spacelike components of the tangent vector $l$ relative to the triad $e_{\underline{i}}$ :

$$
l^{\underline{i}}=-\left(l . e_{\underline{i}}\right) .
$$

Then $\phi_{u}$ may be obtained as a function of the direction of each ray by inserting $l=(l . u) u+l^{\underline{i}} e_{\underline{i}}$ and $l^{\prime}=\left(l^{\prime} . u\right) u+l^{\prime} \underline{j} e_{\underline{j}}$ into Eq. (4.1). This procedure may be very useful when it is necessary to take into account the attitude of the space station on which the measurements are performed, as in models RAMOD for Gaia (see, e.g., de Felice et al. 2006).

2 . However, the angular distance may also be directly obtained by carrying out the calculation of $\sin ^{2} \phi_{u} / 2$ in the chosen reference frame $\left(x^{\alpha}\right)$ without introducing any tetrad. This point of view is close to the spirit of GREM model elaborated by the Dresden group working on Gaia (see Klioner 2003). It is the point of view chosen here. 
Throughout this paper we assume that space-time may be covered by some global quasi-Galilean coordinate system $x^{\alpha}=\left(x^{0}, \boldsymbol{x}\right)$ in which the metric is written as

$$
g_{\mu \nu}=\eta_{\mu \nu}+h_{\mu \nu}, \quad \eta_{\mu \nu}=\operatorname{diag}(1,-1,-1,-1)
$$

and we denote by $x_{e}=\left(x_{e}^{0}, \boldsymbol{x}_{e}\right)$ and $x_{e^{\prime}}=\left(x_{e^{\prime}}^{0}, \boldsymbol{x}_{e^{\prime}}\right)$ the points where $\Gamma$ and $\Gamma^{\prime}$ are emitted, respectively. In what follows, the coordinate system may be identified with the BCRS recommended by IAU2000 resolutions.

We use the vector notations $\boldsymbol{a}=\left(a^{1}, a^{2}, a^{3}\right)=\left(a^{i}\right)$ and $\underline{\boldsymbol{c}}=\left(c_{1}, c_{2}, c_{3}\right)=\left(c_{i}\right)$. Then, given $\boldsymbol{a}, \boldsymbol{b}$ and $\underline{\boldsymbol{c}}$, we put $\boldsymbol{a} . \boldsymbol{b}=a^{i} b^{i}$, and $\boldsymbol{a} . \underline{\boldsymbol{c}}=a^{i} c_{i}$, the Einstein convention being systematically used for repeated indices.

The zeroth-order direction in which $\Gamma$ is seen by an observer $\mathcal{O}(U)$ at rest relative to the coordinate system $\left(x^{\alpha}\right)$ passing through $x_{o}$ is defined as the vector

$$
\boldsymbol{N}=\left(N^{i}\right), \quad N^{i}=\frac{x_{e}^{i}-x_{o}^{i}}{\left|\boldsymbol{x}_{e}-\boldsymbol{x}_{o}\right|} .
$$

Of course, an analogous vector $\boldsymbol{N}^{\prime}$ may be defined for $\Gamma^{\prime}$. We shall call $\phi_{U}^{(0)}$ the angle between $\boldsymbol{N}$ and $\boldsymbol{N}^{\prime}$.

We put

$$
\boldsymbol{\beta}=\left(\beta^{i}\right), \quad \beta^{i}=\frac{d x^{i}}{d x^{0}}, \quad \beta^{2}=\delta_{i j} \beta^{i} \beta^{i},
$$

where the derivatives $d x^{i} / d x^{0}$ are taken along the worldline of $\mathcal{O}(u)$.

In the presence of gravity, the ratio $\left(l_{i} / l_{0}\right)_{x_{o}}$ is slightly different from $N^{i}$. As long as we may assume that there exists one and only one null geodesic between a source located at $\boldsymbol{x}_{e}$ and $x_{o}$, the difference $\left(l_{i} / l_{0}\right)_{x_{o}}-N^{i}$ is a function of $\boldsymbol{x}_{e}, t_{o}$ and $\boldsymbol{x}_{o}$. So we define the (gravitational) deflection vector as

$$
\underline{\boldsymbol{\lambda}}\left(\boldsymbol{x}_{e}, t_{o}, \boldsymbol{x}_{o}\right)=\left(\lambda_{i}\left(\boldsymbol{x}_{e}, t_{o}, \boldsymbol{x}_{o}\right)\right), \quad \lambda_{i}\left(\boldsymbol{x}_{e}, t_{o}, \boldsymbol{x}_{o}\right)=\left(\frac{l_{i}}{l_{0}}\right)_{x_{o}}-N^{i} .
$$

It is shown in Teyssandier \& Le Poncin-Lafitte (2006) that the angular distance $\phi_{u}$ is determined by the equation

$$
\sin ^{2} \frac{\phi_{u}}{2}=K_{u / U}\left(\mathcal{E}, \mathcal{E}^{\prime}\right) \sin ^{2} \frac{\phi_{U}}{2}
$$

where $K_{u / U}\left(\mathcal{E}, \mathcal{E}^{\prime}\right)$ is given by

$$
K_{u / U}\left(\mathcal{E}, \mathcal{E}^{\prime}\right)=\frac{1}{1+h_{00}} \frac{1-\beta^{2}+h_{00}+2 h_{0 k} \beta^{k}+h_{k l} \beta^{k} \beta^{l}}{[1+\boldsymbol{\beta} \cdot(\boldsymbol{N}+\underline{\boldsymbol{\lambda}})]\left[1+\boldsymbol{\beta} \cdot\left(\boldsymbol{N}^{\prime}+\underline{\boldsymbol{\lambda}}^{\prime}\right)\right]}
$$

and $\phi_{U}$ is the angular distance between $\mathcal{E}$ and $\mathcal{E}^{\prime}$ as measured by $\mathcal{O}(U)$. It may be shown that

$$
\begin{aligned}
\sin ^{2} \frac{\phi_{U}}{2}= & \left(1+h_{00}\right)\left[\sin ^{2} \frac{\phi_{U}^{(0)}}{2}+\frac{1}{2}\left(\underline{\boldsymbol{\lambda}}^{\prime}-\underline{\boldsymbol{\lambda}}\right) \cdot\left(\boldsymbol{N}^{\prime}-\boldsymbol{N}\right)-\frac{1}{4} k^{i j}\left(N^{\prime i}-N^{i}\right)\left(N^{\prime j}-N^{j}\right)\right. \\
& \left.+\frac{1}{4}\left(\underline{\boldsymbol{\lambda}}^{\prime}-\underline{\boldsymbol{\lambda}}\right)^{2}-\frac{1}{2} k^{i j}\left(\lambda_{i}^{\prime}-\lambda_{i}\right)\left(N^{\prime j}-N^{j}\right)-\frac{1}{4} k^{i j}\left(\lambda_{i}^{\prime}-\lambda_{i}\right)\left(\lambda_{j}^{\prime}-\lambda_{j}\right)\right]
\end{aligned}
$$

with

$$
k^{i j}=g^{i j}-\eta^{i j}=-h_{i j}+\eta^{\alpha \beta} h_{i \alpha} h_{j \beta}+O\left(h^{3}\right) .
$$


It is clear that the factor $K_{u / U}\left(\mathcal{E}, \mathcal{E}^{\prime}\right)$ describes the aberration due to the motion of the observer $\mathcal{O}(u)$ relative to the observer $\mathcal{O}(U)$.

Equations (4.6) and (4.8) show that the angular separation $\phi_{u}$ is theoretically determined when the deflection vector $\underline{\boldsymbol{\lambda}}$ is known. Let us emphasize that Eqs. (4.6)-(4.8) are rigorous.

Two kinds of procedures are currently available to determine the deflection vector $\underline{\boldsymbol{\lambda}}\left(\boldsymbol{x}_{e}, t_{o}, \boldsymbol{x}_{o}\right)$.

1. One can try to solve the differential equations satisfied by the null geodesics: this method works well when the rays are coming from infinity and can be extended to rays emitted at a finite distance by some adequate procedure (see, e.g., Brumberg 1991). This method is summarized in Sections 5 and 6 for rays emitted at infinity within the weak-field, linearized approximation.

2. One can also use procedures avoiding the integration of geodesic equations. These methods are particularly convenient when the sources of light rays and the observer are located at a finite distance. They are also more easy to extend to the higher orders of approximation. Some results are briefly presented in Sections 7 and 8 .

At the level of accuracy required by Gaia or SIM missions, it is sufficient to know $\underline{\boldsymbol{\lambda}}\left(\boldsymbol{x}_{e}, t_{o}, \boldsymbol{x}_{o}\right)$ within the weak-field, linearized approximation in almost all the practical cases (see, e.g., Klioner 2003). The only known exception is the very special case of a ray grazing giant planets like Jupiter or Saturn (see Section 8). So we shall content ourselves with the linear approximation in the two next sections.

\section{Equations of null geodesics}

A geodesic of $\left(\mathcal{V}_{4}, g\right)$ is a parametrized curve $x^{\rho}(\zeta)$ satisfying the variational principle

$$
\delta \int \frac{1}{2} g_{\alpha \beta}\left(x^{\rho}(\zeta)\right) l^{\alpha} l^{\beta} d \zeta=0, \quad l^{\alpha}=\frac{d x^{\alpha}(\zeta)}{d \zeta} .
$$

Condition (5.1) means that any geodesic $x^{\rho}(\zeta)$ satisfies the following Euler-Lagrange equations

$$
\frac{d l_{\mu}}{d \zeta}=\frac{1}{2} \frac{\partial h_{\alpha \beta}}{\partial x^{\mu}}\left(x^{\rho}(\zeta)\right) l^{\alpha} l^{\beta}, \quad l_{\mu}=g_{\mu \nu} l^{\nu} .
$$

Of course, any solution to Eqs. (5.2) representing a null geodesic has to fulfil the condition (3.1).

We assume henceforth that the gravitational perturbation $h_{\mu \nu}$ can be written as a power series in the gravitational constant $G$

$$
h_{\mu \nu}(x, G)=\sum_{n=1}^{\infty} G^{n} g_{\mu \nu}^{(n)}(x) .
$$

So the solutions to Eqs. (5.2) and (3.1) may be written as

$$
x^{\rho}(\zeta)=x_{(0)}^{\rho}(\zeta)+\sum_{n=1}^{\infty} G^{n} x_{(n)}^{\rho}(\zeta),
$$

which implies for the covariant components of the tangent vector

$$
l_{\mu}(\zeta)=l_{\mu}^{(0)}(\zeta)+\sum_{n=1}^{\infty} G^{n} l_{\mu}^{(n)}(\zeta)
$$

and for the deflection vector

$$
\underline{\boldsymbol{\lambda}}\left(\boldsymbol{x}_{e}, t_{o}, \boldsymbol{x}_{o}\right)=\sum_{n=1}^{\infty} G^{n} \underline{\boldsymbol{\lambda}}^{(n)}\left(\boldsymbol{x}_{e}, t_{o}, \boldsymbol{x}_{o}\right) .
$$


After substituting for $l_{\mu}$ from Eq. (5.5) into Eqs. (5.2) and (3.1), it is easy to see that $l_{\mu}^{(0)}$ is a constant:

$$
l_{\mu}^{(0)}=K_{\mu}, \quad K_{\mu}=\text { const },
$$

where the $K_{\mu}$ have to fulfil the condition

$$
\eta_{\alpha \beta} K^{\alpha} K^{\beta}=0, \quad K^{\alpha}=\eta^{\alpha \mu} K_{\mu} .
$$

For any null geodesic of 0th-order direction $-\boldsymbol{N}$ arriving at $x_{o}$, the constants $K^{i}$ may be chosen so that $K^{i}=-N^{i}$. Hence $K^{0}=1$ as a consequence of (5.8). Denoting by $\zeta_{o}$ the (arbitrary) value of $\zeta$ at point $x_{o}$, the zeroth-order parametric equations of the null geodesic are then

$$
x_{(0)}^{0}(\zeta)=\zeta-\zeta_{o}+x_{o}^{0}, \quad \boldsymbol{x}_{(0)}(\zeta)=-\left(\zeta-\zeta_{o}\right) \boldsymbol{N}+\boldsymbol{x}_{o} .
$$

Taking (5.7) and (5.9) into account, and then adopting the notation

$$
f_{, \mu}(x)=\frac{\partial f}{\partial x^{\mu}}
$$

for the partial differentiation of any function of $x$, the differential equation satisfied by $l_{\mu}^{(1)}$ may be written as

$$
\frac{d l_{\mu}^{(1)}}{d \zeta}=\frac{1}{2}\left[g_{00, \mu}^{(1)}-2 g_{0 k, \mu}^{(1)} N^{k}+g_{k l, \mu}^{(1)} N^{k} N^{l}\right]_{x_{(0)}(\zeta)} .
$$

A straightforward calculation shows that Eq. (3.1) reduces to a constraint equation which determines $l_{0}^{(1)}$ :

$$
l_{0}^{(1)}=\frac{1}{2}\left[g_{00}^{(1)}-2 g_{0 k}^{(1)} N^{k}+g_{k l}^{(1)} N^{k} N^{l}+2 N^{l} l_{l}^{(1)}\right]_{x_{(0)}(\zeta)} .
$$

As a consequence, the first-order term $\lambda_{i}^{(1)}$ is given by

$$
\lambda_{i}^{(1)}=\left(\delta_{i}^{j}-N^{i} N^{j}\right) l_{j}^{(1)}-\frac{1}{2} N^{i}\left[g_{00}^{(1)}-2 g_{0 k}^{(1)} N^{k}+g_{k l}^{(1)} N^{k} N^{l}\right] .
$$

Thus the deflection vector at $x_{o}$ is completely determined when the three spacelike covariant components $l_{i}^{(1)}$ are known. The next section is devoted to the expression of $l_{i}^{(1)}$ and $\lambda_{i}^{(1)}$ for a light ray emitted at infinity.

\section{Light rays coming from infinity}

In this section, we suppose that the gravitational potentials $h_{\mu \nu}$ and their first derivatives tend to zero when $|\boldsymbol{x}| \rightarrow \infty$. Assuming that the light ray is emitted at infinity in a direction $-\boldsymbol{N}$, an integration of Eqs. (5.11) yields for the value of $l_{j}^{(1)}$ at $x_{o}$

$$
l_{j}^{(1)}=\frac{1}{2} \int_{-\infty}^{\zeta_{o}}\left[g_{00, j}^{(1)}-2 g_{0 k, j}^{(1)} N^{k}+g_{k l, j}^{(1)} N^{k} N^{l}\right]_{x_{(0)}(\zeta)} d \zeta
$$

the integral being taken along the zeroth-order straight line defined by Eqs. (5.9).

We consider that the Solar System is an isolated system constituted by N slowly moving, self gravitating bodies $A(A=1,2, \ldots, N)$. The integrals involved in Eqs. (6.1) have been studied using the technique of retarded potentials (see, e.g., Kopeikin \& Schäfer 1999 and Kopeikin et al. 2006). However, it is generally estimated that the deflections due to the retarded effects are less than $1 \mu$ as and can therefore be currently neglected. 
So we may content ourselves with using the parametrized post-Newtonian (1PPN) approximation developed in Klioner \& Soffel (2004), which is a natural extension of the IAU2000 metric. Since a consistent treatment of light propagation requires to retain only the metric truncated at $1 / c^{3}$, we take

$$
g_{00}^{(1)}=-\frac{2 w}{c^{2}}+O(4), \quad\left(g_{0 k}^{(1)}\right)=(\gamma+1) \frac{2 \boldsymbol{w}}{c^{3}}+O(5), \quad g_{k l}^{(1)}=-\gamma \frac{2 w}{c^{2}} \delta_{k l}+O(4),
$$

where

$$
\begin{aligned}
& w\left(x^{0}, \boldsymbol{x}\right)=\sum_{A=1}^{N} w_{A}\left(x^{0}, \boldsymbol{x}\right), \quad w_{A}\left(x^{0}, \boldsymbol{x}\right)=\int \frac{\rho_{A}\left(x^{0}, \boldsymbol{x}_{A}^{\prime}\right)}{\left|\boldsymbol{x}-\boldsymbol{x}_{A}^{\prime}\right|} d^{3} \boldsymbol{x}_{A}^{\prime}, \\
& \boldsymbol{w}\left(x^{0}, \boldsymbol{x}\right)=\sum_{A=1}^{N} \boldsymbol{w}_{A}\left(x^{0}, \boldsymbol{x}\right), \quad \boldsymbol{w}_{A}\left(x^{0}, \boldsymbol{x}\right)=\int \frac{\rho_{A}\left(x^{0}, \boldsymbol{x}_{A}^{\prime}\right) \boldsymbol{v}_{A}^{\prime}\left(x^{0}, \boldsymbol{x}_{A}^{\prime}\right)}{\left|\boldsymbol{x}-\boldsymbol{x}_{A}^{\prime}\right|} d^{3} \boldsymbol{x}_{A}^{\prime},
\end{aligned}
$$

$\rho_{A}$ and $\boldsymbol{v}_{A}^{\prime}$ being the rest-mass density and the velocity-field of the matter constituting the body $A$, respectively.

A rough estimate shows that the Sun and the planets may be considered as axially symmetric bodies slowly spinning about their axis of symmetry. Moreover, the multipole expansion of the gravitomagnetic potentials $\boldsymbol{w}_{A}\left(x^{0}, \boldsymbol{x}\right)$ may be completely neglected. We may content ourselves with the following expressions for $w_{A}\left(x^{0}, \boldsymbol{x}\right)$ and $\boldsymbol{w}_{A}\left(x^{0}, \boldsymbol{x}\right)$ :

$$
\begin{gathered}
w_{A}\left(x^{0}, \boldsymbol{x}\right)=\frac{M_{A}}{\left|\boldsymbol{x}-\boldsymbol{x}_{A}\right|}\left\{1-J_{1 A} R_{A} \frac{\boldsymbol{k}_{A} \cdot\left(\boldsymbol{x}-\boldsymbol{x}_{A}\right)}{\left|\boldsymbol{x}-\boldsymbol{x}_{A}\right|^{2}}\right. \\
\left.-J_{2 A} R_{A}^{2} \frac{3\left[\boldsymbol{k}_{A} \cdot\left(\boldsymbol{x}-\boldsymbol{x}_{A}\right)\right]^{2}-\left(\boldsymbol{x}-\boldsymbol{x}_{A}\right)^{2}}{2\left|\boldsymbol{x}-\boldsymbol{x}_{A}\right|^{4}}+\cdots\right\} \\
\boldsymbol{w}_{A}\left(x^{0}, \boldsymbol{x}\right)=w_{A}\left(x^{0}, \boldsymbol{x}\right) \boldsymbol{v}_{A}\left(x^{0}\right)+\frac{\boldsymbol{S}_{A} \times\left(\boldsymbol{x}-\boldsymbol{x}_{A}\right)}{2\left|\boldsymbol{x}-\boldsymbol{x}_{A}\right|^{3}}+\cdots,
\end{gathered}
$$

where, for each body $A, \boldsymbol{x}_{A}$ is the point on the axis of symmetry supporting the multipole distribution, $M_{A}$ the mass, $J_{1 A}, J_{2 A}, \ldots$ the mass-multipole moments, $R_{A}$ the equatorial radius, $\boldsymbol{k}_{A}$ the unit vector on the axis of symmetry, $\boldsymbol{S}_{A}$ the intrinsic angular momentum $\left(\boldsymbol{S}_{A}=S_{A} \boldsymbol{k}_{A}\right)$ and $\boldsymbol{v}_{A}=c d \boldsymbol{x}_{A} / d x^{0}\left(\boldsymbol{x}_{A}\right.$ is a function of $\left.x^{0}\right)$.

The insertion of Eqs. (6.5) and (6.6) in Eqs. (6.1) leads to expressions impossible to calculate analytically if the full variation in time of $\boldsymbol{x}_{A}$ is taken into account. However, one can obtain a correct estimate of integrals (6.1) assuming that each body $A$ is fixed at its position $\boldsymbol{x}_{A o}$ at the moment $t_{A o}$ of the closest approach of the body and the photon (Klioner \& Kopeikin 1992 and Klioner 2003).

Let $P_{A o}$ be the projection of the position of point $\boldsymbol{x}_{A o}$ on the straight line parallel to $\boldsymbol{N}$ passing through $\boldsymbol{x}_{o}$. Putting

$$
\boldsymbol{b}_{A o}=\boldsymbol{x}_{P_{A o}}-\boldsymbol{x}_{A o}, \quad \boldsymbol{p}_{A o}=\frac{\boldsymbol{b}_{A o}}{b_{A o}}, \quad \boldsymbol{q}_{A o}=\boldsymbol{p}_{A o} \times \boldsymbol{N}, \quad \boldsymbol{n}_{A o}=\frac{\boldsymbol{x}_{o}-\boldsymbol{x}_{A o}}{\left|\boldsymbol{x}_{o}-\boldsymbol{x}_{A o}\right|}
$$

and denoting by $\alpha_{A o}$ the angle between $-\boldsymbol{n}_{A o}$ and $\boldsymbol{N}$, the deflection vector is given by

$$
\underline{\boldsymbol{\lambda}}^{(1)}\left(\boldsymbol{N}, \boldsymbol{x}_{o}\right)=\sum_{A=1}^{N} \underline{\boldsymbol{\lambda}}_{M_{A}}^{(1)}+\underline{\boldsymbol{\lambda}}_{J_{1 A}}^{(1)}+\underline{\boldsymbol{\lambda}}_{J_{2 A}}^{(1)}+\underline{\boldsymbol{\lambda}}_{S_{A}}^{(1)}+\cdots
$$


where

$$
\begin{aligned}
\underline{\boldsymbol{\lambda}}_{M_{A}}^{(1)}= & (\gamma+1) \frac{M_{A}}{c^{2} b_{A o}}\left[\sin \alpha_{A o} \boldsymbol{N}+\left(1+\cos \alpha_{A o}\right) \boldsymbol{p}_{A o}\right]+\cdots, \\
\underline{\boldsymbol{\lambda}}_{J_{1 A}}^{(1)}= & (\gamma+1) \frac{M_{A}}{c^{2} b_{A o}} J_{1 A} \frac{R_{A}}{b_{A o}}\left(1+\cos \alpha_{A o}\right)\left[\left(\boldsymbol{k}_{A o} \cdot \boldsymbol{q}_{A o}\right) \boldsymbol{q}_{A o}-\left(\boldsymbol{k}_{A o} \cdot \boldsymbol{p}_{A o}\right) \boldsymbol{p}_{A o}\right]+\cdots, \\
\underline{\boldsymbol{\lambda}}_{J_{2 A}}^{(1)}= & (\gamma+1) \frac{M_{A}}{c^{2} b_{A o}} J_{2 A}\left(\frac{R_{A}}{b_{A o}}\right)^{2}\left(1+\cos \alpha_{A o}\right)\left\{\left[\left(\boldsymbol{k}_{A o} \cdot \boldsymbol{q}_{A o}\right)^{2}-\left(\boldsymbol{k}_{A o} \cdot \boldsymbol{p}_{A o}\right)^{2}\right] \boldsymbol{p}_{A o}\right. \\
& \left.+2\left(\boldsymbol{k}_{A o} \cdot \boldsymbol{p}_{A o}\right)\left(\boldsymbol{k}_{A o} \cdot \boldsymbol{q}_{A o}\right) \boldsymbol{q}_{A o}\right\}+\cdots, \\
\underline{\boldsymbol{\lambda}}_{S_{A}}^{(1)}= & (\gamma+1) \frac{S_{A}}{c^{3} b_{A o}^{2}}\left(1+\cos \alpha_{A o}\right)\left[\left(\boldsymbol{k}_{A o} \cdot \boldsymbol{q}_{A o}\right) \boldsymbol{p}_{A o}+\left(\boldsymbol{k}_{A o} \cdot \boldsymbol{p}_{A o}\right) \boldsymbol{q}_{A o}\right]+\cdots
\end{aligned}
$$

$\boldsymbol{k}_{A o}$ being the vector $\boldsymbol{k}$ at instant $t_{A o}$ and $+\cdots$ standing for terms giving negligible contributions at the $1 \mu$ as level (case, e.g., of the translational gravitomagnetic terms).

The contributions of the mass-multipole moments $J_{1}$ and $J_{2}$ are analyzed in several works (see, e.g., Kopeikin \& Makarov 2007 and Refs. therein). The contributions of the other moments $J_{n}$ are thoroughly calculated in Le Poncin-Lafitte \& Teyssandier (2008).

A test of the deflection due to the mass quadrupole moment of Jupiter, say $\left(J_{2}\right)_{J u p}$, was proposed in Crosta \& Mignard (2006) in the context of the Gaia mission (project GAREX). This test seems feasible since the maximum quadrupolar deflection predicted for a ray grazing Jupiter amounts to $\left(\widehat{\delta}_{J_{2}}\right)_{\text {Jup }}=240 \mu$ as.

A general account of the effects relevant to a $1 \mu$ as accuracy is given in Klioner (2003). It must be noted that the deflection due to the gravitomagnetic term $\underline{\boldsymbol{\lambda}}_{S_{A}}^{(1)}$ is less than $1 \mu$ as for the Sun and the giant planets.

\section{Sources at a finite distance}

Let us outline the new methods developed in Le Poncin-Lafitte et al. (2004) and Teyssandier \& Le Poncin-Lafitte (2008). It follows from these works that the deflection vector is given by

$$
\lambda_{i}=-\frac{c \partial \mathcal{T}_{r}}{\partial x_{o}^{i}}\left[1-\frac{c \partial \mathcal{T}_{r}}{\partial x_{o}^{0}}\right]^{-1}-N^{i},
$$

where $\mathcal{T}_{r}$ is the so-called reception time transfer function giving the travel time of a photon as a function of the spatial position of the emitter $\boldsymbol{x}_{e}$, the instant of reception $x_{o}^{0}$ and the spatial position $\boldsymbol{x}_{o}$ of the observer, so that $x_{o}^{0}-x_{e}^{0}$ can be written as

$$
x_{o}^{0}-x_{e}^{0}=c \mathcal{T}_{r}\left(\boldsymbol{x}_{e}, x_{o}^{0}, \boldsymbol{x}_{o}\right) .
$$

Moreover, assuming that the reception time transfer function may be expanded as

$$
c \mathcal{T}_{r}\left(\boldsymbol{x}_{e}, x_{o}^{0}, \boldsymbol{x}_{o}\right)=\left|\boldsymbol{x}_{e}-\boldsymbol{x}_{o}\right|+c \sum_{n=1}^{\infty} G^{n} \mathcal{T}_{r}^{(n)}\left(\boldsymbol{x}_{e}, x_{o}^{0}, \boldsymbol{x}_{o}\right)
$$

it is shown that each perturbation term $\mathcal{T}_{r}^{(n)}\left(\boldsymbol{x}_{e}, x_{o}^{0}, \boldsymbol{x}_{o}\right)$ is given by an integral over the null geodesic of Minkowski space-time having $-\boldsymbol{N}$ as spatial direction and arriving at $\left(x_{o}^{0}, \boldsymbol{x}_{o}\right)$, that is the curve defined by the parametric equations

$$
z_{-}(\zeta)=\left(x_{o}^{0}-\zeta\left|\boldsymbol{x}_{e}-\boldsymbol{x}_{o}\right|, \boldsymbol{x}_{o}+\zeta\left|\boldsymbol{x}_{e}-\boldsymbol{x}_{o}\right| \boldsymbol{N}\right) .
$$


One thus recovers the well-known expression for the first-order term $\mathcal{T}_{r}^{(1)}$ :

$$
c \mathcal{T}_{r}^{(1)}\left(\boldsymbol{x}_{e}, x_{o}^{0}, \boldsymbol{x}_{o}\right)=\frac{1}{2}\left|\boldsymbol{x}_{e}-\boldsymbol{x}_{o}\right| \int_{0}^{1}\left[g_{(1)}^{00}+2 N^{i} g_{(1)}^{0 i}+N^{i} N^{j} g_{(1)}^{i j}\right]_{z_{-}(\zeta)} d \zeta .
$$

The higher-order terms $\mathcal{T}_{r}^{(n)}$ are very complicated. We shall content ourselves here with giving the expression of $c \mathcal{T}^{(2)}$ for a stationary gravitational field. In this case indeed, the quantities $\mathcal{T}_{r}^{(n)}$ do not depend on the instant of reception $t_{r}$. Then

$$
\begin{aligned}
c \mathcal{T}^{(2)}\left(\boldsymbol{x}_{e}, \boldsymbol{x}_{o}\right)= & \frac{1}{2}\left|\boldsymbol{x}_{e}-\boldsymbol{x}_{o}\right| \int_{0}^{1}\left\{\left[g_{(2)}^{00}+2 N^{i} g_{(2)}^{0 i}+N^{i} N^{j} g_{(2)}^{i j}\right]_{\boldsymbol{z}_{-}(\zeta)}\right. \\
& +2\left[g_{(1)}^{0 i}+N^{j} g_{(1)}^{i j}\right]_{\boldsymbol{z}_{-}(\zeta)} \frac{c \partial \mathcal{T}^{(1)}}{\partial x^{i}}\left(\boldsymbol{z}_{-}(\zeta), \boldsymbol{x}_{o}\right) \\
& \left.+\eta^{i j}\left[\frac{c \partial \mathcal{T}^{(1)}}{\partial x^{i}} \frac{c \partial \mathcal{T}^{(1)}}{\partial x^{j}}\right]_{\left(\boldsymbol{z}_{-}(\zeta), \boldsymbol{x}_{o}\right)}\right\} d \zeta
\end{aligned}
$$

where $\boldsymbol{z}_{-}(\zeta)=\boldsymbol{x}_{o}+\zeta\left|\boldsymbol{x}_{e}-\boldsymbol{x}_{o}\right| \boldsymbol{N}$.

\section{Application to a static spherically symmetric metric}

Let us consider the family of static, spherically symmetric metrics written in the form $d s^{2}=\left(1-\frac{2 G M}{c^{2} r}+2 \beta \frac{G^{2} M^{2}}{c^{4} r^{2}}+\ldots\right)\left(d x^{0}\right)^{2}-\left(1+2 \gamma \frac{G M}{c^{2} r}+\frac{3}{2} \delta \frac{G^{2} M^{2}}{c^{4} r^{2}}+\cdots\right) \delta_{i j} d x^{i} d x^{j}$,

where $\beta$ and $\gamma$ are the usual post-Newtonian parameters and $\delta$ is a supplementry postpost-Newtonian parameter $(\gamma=\beta=\delta=1$ in general relativity). It follows from Eqs. (7.5) and (7.6) that (see Teyssandier \& Le Poncin-Lafitte 2008)

$$
\begin{aligned}
\mathcal{T}\left(\boldsymbol{x}_{e}, \boldsymbol{x}_{o}\right)= & \frac{\left|\boldsymbol{x}_{e}-\boldsymbol{x}_{o}\right|}{c}+(\gamma+1) \frac{G M}{c^{3}} \ln \left[\frac{\left|\boldsymbol{x}_{e}\right|+\left|\boldsymbol{x}_{o}\right|+\left|\boldsymbol{x}_{e}-\boldsymbol{x}_{o}\right|}{\left|\boldsymbol{x}_{e}\right|+\left|\boldsymbol{x}_{o}\right|-\left|\boldsymbol{x}_{e}-\boldsymbol{x}_{o}\right|}\right] \\
& +\frac{G^{2} M^{2}}{c^{5}} \frac{\left|\boldsymbol{x}_{e}-\boldsymbol{x}_{o}\right|}{\left|\boldsymbol{x}_{e}\right|\left|\boldsymbol{x}_{o}\right|}\left[\kappa \frac{\arccos \left(\boldsymbol{n}_{e} \cdot \boldsymbol{n}_{o}\right)}{\sqrt{1-\left(\boldsymbol{n}_{e} \cdot \boldsymbol{n}_{o}\right)^{2}}}-\frac{(1+\gamma)^{2}}{1+\boldsymbol{n}_{e} \cdot \boldsymbol{n}_{o}}\right]+O\left(c^{-7}\right),
\end{aligned}
$$

where

$$
\boldsymbol{n}_{e}=\frac{\boldsymbol{x}_{e}}{\left|\boldsymbol{x}_{e}\right|}, \quad \boldsymbol{n}_{o}=\frac{\boldsymbol{x}_{o}}{\left|\boldsymbol{x}_{o}\right|}, \quad \kappa=\frac{1}{4}(8-4 \beta+8 \gamma+3 \delta) .
$$

Equation (8.2) generalizes a result given in Brumberg (1987) for general relativity $(\kappa=15 / 4)$.

Substituting for $\mathcal{T}$ from Eq. (8.2) into Eqs. (7.1) yields the deflection vector at point $x_{o}$. Let $P$ be the foot of the perpendicular drawn from origin $O$ to the straight line parallel to $\boldsymbol{N}$ passing through $\boldsymbol{x}_{o}$. Putting

$$
b_{(0)}=\left|\boldsymbol{x}_{P}\right|, \quad \boldsymbol{p}=\frac{\boldsymbol{x}_{P}}{b_{(0)}},
$$

and then noting that the zeroth-order impact parameter $b_{(0)}=r_{o} \sin \alpha_{o}$, where $\alpha_{o}$ is the angle between $-\boldsymbol{n}_{o}$ and $\boldsymbol{N}$, we get in the case of a source located at infinity:

$$
\begin{aligned}
\underline{\boldsymbol{\lambda}}= & \frac{G M}{c^{2} b_{(0)}}\left\{\left[(\gamma+1) \sin \alpha_{o}+\frac{G M}{c^{2} b_{(0)}}\left(1+\cos \alpha_{o}\right)\left[2 \kappa \sin ^{2} \frac{\alpha_{o}}{2}-(\gamma+1)^{2}\right]\right] \boldsymbol{N}\right. \\
& \left.+\left[(\gamma+1)\left(1+\cos \alpha_{o}\right)+\frac{G M}{c^{2} b_{(0)}}\left[\kappa\left(\pi-\alpha_{o}+\frac{1}{2} \sin 2 \alpha_{o}\right)-\mathcal{C}^{(2)}\right]\right] \boldsymbol{p}\right\}
\end{aligned}
$$


where

$$
\mathcal{C}^{(2)}=(\gamma+1)^{2} \frac{\left(1+\cos \alpha_{o}\right)^{2}}{\sin \alpha_{o}} .
$$

The contribution due to the second-order term $\mathcal{C}^{(2)}$ may compare with first-order contributions when $\alpha_{o}$ becomes sufficiently small. Indeed, this term yields a deflection of $16.1 \mu$ as for a light ray grazing Jupiter and observed at a distance of 6 AU (for $\gamma=1$ ). It is exactly the result obtained in Zschocke \& Klioner (2009) by a different calculation.

\section{Some concluding remarks}

The first-order approximation set out in Sections 5 and 6 is generally sufficient for space astrometric missions like Gaia or SIM. However, the special case of light rays grazing the surface of a giant planet and some highly precise tests of general relativity in foreseeable future will require calculations within the post-linear regime. The method presented in Sections 7 and 8 is able to vie with the integration of geodesic equations in the treatment of these problems. This procedure directly yields the deflection vector for a family of parametrized post-post-Newtonian spherically symmetric metrics.

A more radical improvement of the modelizations will require to treat the propagation of light in dynamical extensions of the IAU2000 metric including all the possible $c^{-4}$ terms (see, e.g., Minazzoli \& Chauvineau 2009 and Refs. therein). In the longer term, it will probably become necessary to take into account the cosmological background, as it is already outlined in Klioner \& Soffel (2004).

\section{References}

Brumberg, V. A. 1987, Kinematics Phys. Celest. Bodies, 3, 6

Brumberg, V. A. 1991, Essential Relativistic Celestial Mechanics (Bristol: Adam Hilger), p. 200 Crosta, M. T. \& Mignard, F. 2006, Class. Quantum Grav., 23, 4853

de Felice, F., Vecchiato, A., Crosta, M. T., Bucciarelli, B., \& Lattanzi, M. G. 2006, ApJ, 653, 1552

de Jans, C. 1922, Mém. Acad. Roy. Belgique Cl. Sci., 6, pp.1-26; 6, pp. 27-41

Klioner, S. A. \& Kopeikin, S. M. 1992, Astron. J., 104, 897

Klioner, S. A. \& Soffel, M. H. 2000, Phys. Rev. D, 62, 024019

Klioner, S. A. 2003, Astron. J., 125, 1580

Klioner, S. A \& Soffel, M. H. 2004, in: C. Turon, K. S. O'Flaherty, \& M. A. C. Perryman (eds.), Proceedings of the Symposium "The Three-Dimensional Universe with Gaia" (ESA SP-576, 2005), p. 305

Kopeikin, S. M. 1997, J. Math. Phys., 38, 2587

Kopeikin, S. M. \& Schäfer, G. 1999, Phys. Rev. D, 60, 124002

Kopeikin, S. M., Korobkov, P., \& Polnarev, A. 2006, Class. Quantum Gravity, 23, 4299

Kopeikin, S. M. \& Makarov, V. V. 2007, Phys. Rev. D, 75, 062002

Le Poncin-Lafitte C., Linet, B., \& Teyssandier P. 2004, Class. Quantum Gravity, 21, 4463

Le Poncin-Lafitte C., \& Teyssandier P. 2008, Phys. Rev. D, 77, 044029

Minazzoli, O., \& Chauvineau, B. 2009, Phys. Rev. D, 79, 084027

Soffel, M. H. 1989, Relativity in Astrometry, Celestial Mechanics and Geodesy (Berlin: SpringerVerlag)

Teyssandier, P., \& Le Poncin-Lafitte C. 2006, arXiv:gr-qc/0611078

Teyssandier, P., \& Le Poncin-Lafitte C. 2008, Class. Quantum Gravity, 25, 145020

Will, C. M. 1988, Am. J. Phys., 56, 413

Zschocke, S. \& Klioner S. A. 2009, arXiv:astro-ph/09043704 